\title{
The management of metastatic
} radioiodine-refractory differentiated thyroid cancer requires an integrated approach including both directed and systemic therapies

\author{
Shamil D Cooray ${ }^{1}$ and Duncan J Topliss ${ }^{1,2}$ \\ 1Department of Endocrinology and Diabetes, The Alfred, Melbourne, Australia and ${ }^{2}$ Department of \\ Medicine, Monash University, Melbourne, Australia
}

\author{
Correspondence \\ should be addressed \\ to D J Topliss \\ Email \\ D.Topliss@alfred.org.au
}

\section{Summary}

A 58-year-old man with metastatic radioiodine-refractory differentiated thyroid cancer (DTC) presented with left thigh and right flank numbness. He had known progressive and widespread bony metastases, for which he received palliative radiotherapy, and multiple bilateral asymptomatic pulmonary metastases. CT scan and MRI of the spine revealed metastases at right T10-L1 vertebrae with extension into the central canal and epidural disease at T10 and T11 causing cord displacement and canal stenosis but retention of spinal cord signal. Spinal surgery was followed by palliative radiotherapy resulting in symptom resolution. Two months later, sorafenib received approval for use in Australia and was commenced and up-titrated with symptomatic management of mild adverse effects. Follow-up CT scan three months after commencement of sorafenib revealed regression of pulmonary metastases but no evident change in most bone metastases except for an advancing lesion eroding into the right acetabulum. The patient underwent a right total hip replacement, intra-lesional curettage and cementing. After six months of sorafenib therapy, CT scanning showed enlarging liver lesions with marked elevation of serum thyroglobulin. Lenvatinib was commenced and sorafenib was ceased. He now has stable disease with a falling thyroglobulin more than 5 years after metastatic radioiodine-refractory DTC was diagnosed. In DTC, $5 \%$ of distant metastases become radioiodine-refractory, resulting in a median overall survival of 2.5-3.5 years. Tyrosine kinase inhibitor (TKI) therapy has recently been demonstrated to increase progression-free survival in these patients but poses some unique management issues and is best used as part of an integrated approach with directed therapy.

\section{Learning points:}

- Directed therapies may have greater potential to control localised disease and related symptoms when compared to systemic therapies.

- Consider TKI therapy in progressive disease where benefits outweigh risks.

- Active surveillance and timely intervention are required for TKI-related adverse effects.

- There is a need for further research on the clinical application of TKI therapy in advanced DTC, including comparative efficacy, sequencing and identifying responders.

\section{Background}

The incidence of thyroid cancer is increasing. Differentiated thyroid cancer (DTC) is the most common type, making up approximately $98 \%$ of cases. Ten to fifteen percent of patients develop distant metastases and about $5 \%$ of distant metastases become radioiodinerefractory and therefore have a very unfavourable 
prognosis, with a median overall survival of 2.5-3.5 years $(1)$.

Tyrosine kinase inhibitor therapy has recently been demonstrated to increase progression-free survival in these patients but poses some unique management issues. Despite this novel therapy, a prudent approach should integrate both locally directed and systemic therapies.

\section{Case presentation}

A 58-year-old man with metastatic radioiodine-refractory DTC presented with left thigh pain and right flank numbness.

He had known progressive and widespread bony metastases, for which he has received palliative radiotherapy, and multiple bilateral asymptomatic pulmonary metastases. Progression of his bony metastatic disease led to withdrawal from an investigational MEK inhibitor drug trial after seven months. His initial presentation was fourteen years before, after he underwent a hemi-thyroidectomy for a lesion subsequently diagnosed as a Hürthle cell carcinoma, which was complicated by local recurrence after 7 years requiring surgical resection via right vertical hemi-laryngectomy, completion thyroidectomy and radioactive iodine (RAI) ablation. Over this period of follow-up, he received a further course of RAI ablation, a partial left hemi-laryngectomy and adjuvant radiotherapy for a second local recurrence. He has been maintained throughout on suppressive thyroxine therapy.

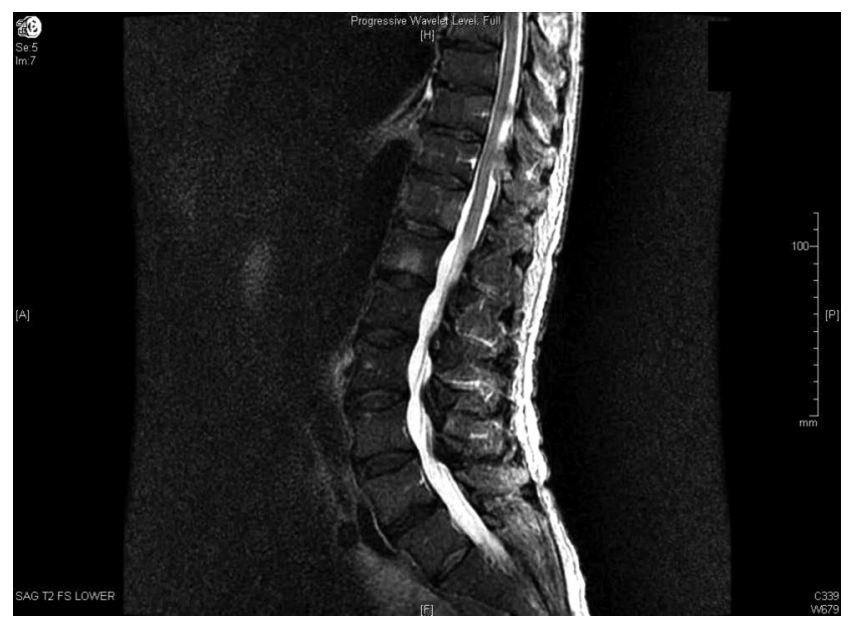

Figure 1

MRI scan of spine revealed epidural disease at T10 and T11 causing cord displacement and canal stenosis but retention of spinal cord signal on a T2 sequence.
He worked full-time as a bus driver but this had been limited recently with a performance status consistent with Eastern Cooperative Oncology Group (ECOG) score of 1.

Examination revealed a husky and weak voice. There were no neck masses. His chest was clear. Abdominal examination did not reveal any specific organomegaly, and liver dullness was normal. Neurological examination revealed diminished sensation over the outer left thigh with loss of light touch and temperature.

\section{Investigation}

CT scan and MRI of the spine revealed metastases at right T10-L1 vertebrae with extension into the central canal and epidural disease at T10 and T11 causing cord displacement and canal stenosis but retention of spinal cord signal (Fig. 1).

\section{Treatment}

Spinal surgery was performed with T8-L2 pedicle screw fixation, T10-T11 right-sided hemilaminectomy and transpedicular debulking of T10-T11 bone metastases. The patient was discharged home after an uncomplicated postoperative course and a week of inpatient rehabilitation. He received post-operative palliative radiotherapy to the thoracolumbar spine (20Gy in 5 fractions to T9L1). Surgery and radiotherapy had good effect with resolution of the patient's right flank pain and right lower limb numbness.

Two months later, sorafenib received approval for use in Australia and was commenced at a dose of $200 \mathrm{mg}$ po BD.

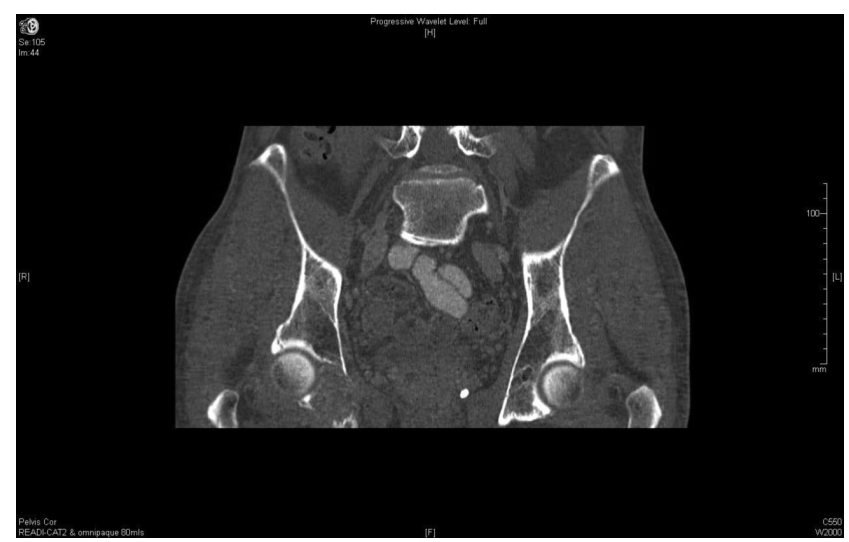

Figure 2

Follow-up CT scan revealed advancement of disease in the right ischial tuberosity with extension into the right acetabulum. 


\section{Outcome and follow-up}

Two weeks after commencement of sorafenib, the patient developed a hand-foot skin reaction, and increased bowel and urinary frequency. Symptoms were persistent but tolerable at the four-week review; thus, the dose was increased to $400 \mathrm{mg}$ po BD. On the full dose, the patient's main adverse effect was diarrhoea, which was managed with prophylactic loperamide.

Follow-up CT scan three months after commencement of sorafenib revealed regression of pulmonary metastases but progression of bony metastases (Fig. 2). A restaging co-registered PET/CT scan in the setting of consideration for right hip/pelvic surgery was conducted (Fig. 3).

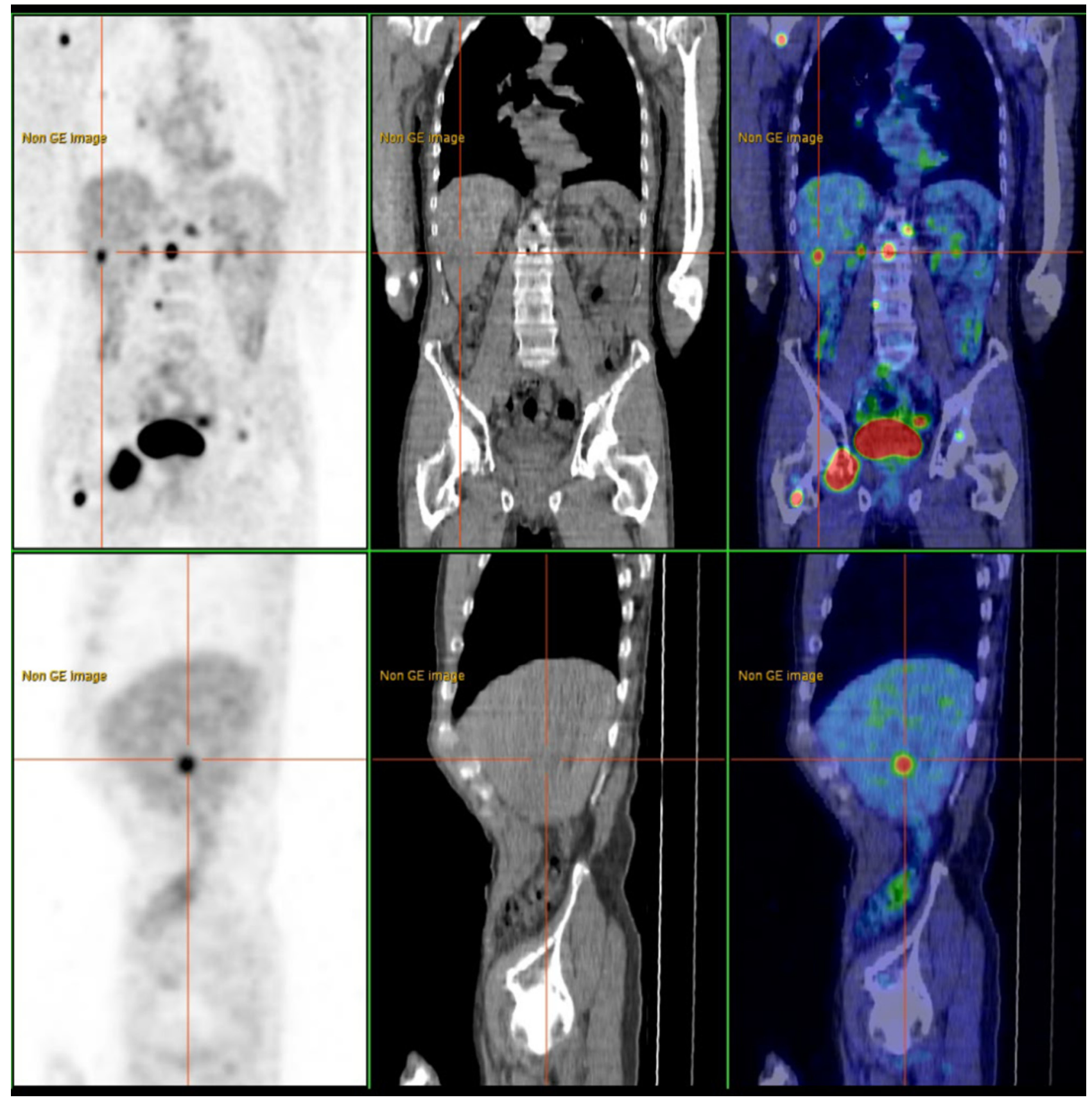

Figure 3

A restaging co-registered PET/CT scan revealed new hepatic metastatic disease and progression of the widespread bony disease. The top row shows PET, $\mathrm{CT}$ and fused cross-sectional coronal views and the bottom row sagittal views demonstrating the new hepatic metastatic disease and bony metastases in the right scapula, right ischium, right proximal femur and lumbar vertebrae. 


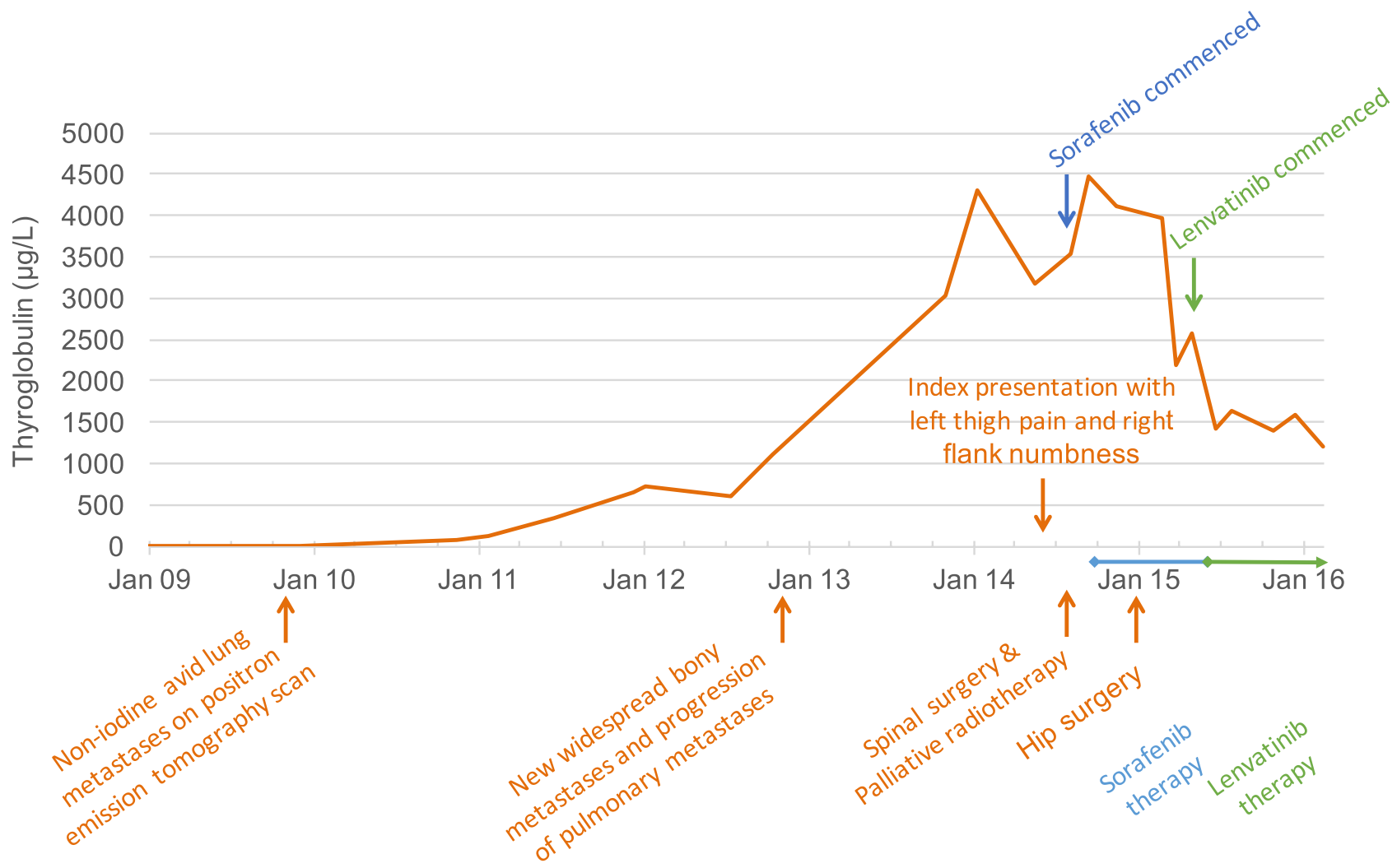

Figure 4

Marked elevation of serum thyroglobulin heralded metastatic disease but is now falling on TKI therapy, observation time $2009-2016$.

The patient underwent a right total hip replacement, intra-lesional curettage and cementing.

After six months of sorafenib therapy, CT scanning showed enlarging liver lesions with marked elevation of serum thyroglobulin (Fig. 4). Lenvatinib was commenced and sorafenib was ceased. He now has stable disease with regression of liver lesions and a falling thyroglobulin more than 5 years after metastatic radioiodine-refractory DTC was diagnosed. Zoledronic acid has been administered to reduce fracture risk from bony metastatic disease. He continues full-time employment.

\section{Discussion}

This case is an example of radioiodine-refractory DTC as per published criteria (2). The management approach to radioiodine-refractory DTC is based on optimally applied conventional therapy before consideration of local and systemic therapies (Fig. 5). Novel systemic therapies should only be considered if disease is progressive, and both clinician and patient agree that benefits are expected to outweigh risks, particularly regarding quality of life (2). If disease is stable, and therefore benefits of any systemic therapy is likely to be outweighed by risks, active surveillance is the recommended approach (2). Tyrosine kinase inhibitors are now considered the firstline systemic therapy (2).

The use of tyrosine kinase inhibitors (TKI) in clinical practice requires an appreciation of their mechanism of action and therefore their adverse effects, which guide their relative contraindications, pre-initiation assessment and ongoing monitoring and management. TKIs target a combination of oncoproteins on vascular endothelial cells and oncogenic signalling pathways in tumour cells crucial for cancer progression (3). They are tumourostatic rather than tumoricidal (4). This distinction is key, as clinical benefit will therefore only be apparent in progressive disease; in disease that is already stable, the patient would likely be exposed to adverse effects with little possibility of benefit.

Some adverse effects of this class are common and manageable, whereas others are serious and potentially fatal (2) (Table 1). Pre-existing risk factors for these adverse effects may serve as relative contraindication to their use. Interestingly, some adverse effects like hypertension are associated with a survival benefit and have been suggested to be a biomarker of successful inhibition of the vascular endothelial growth factor pathway (5). 


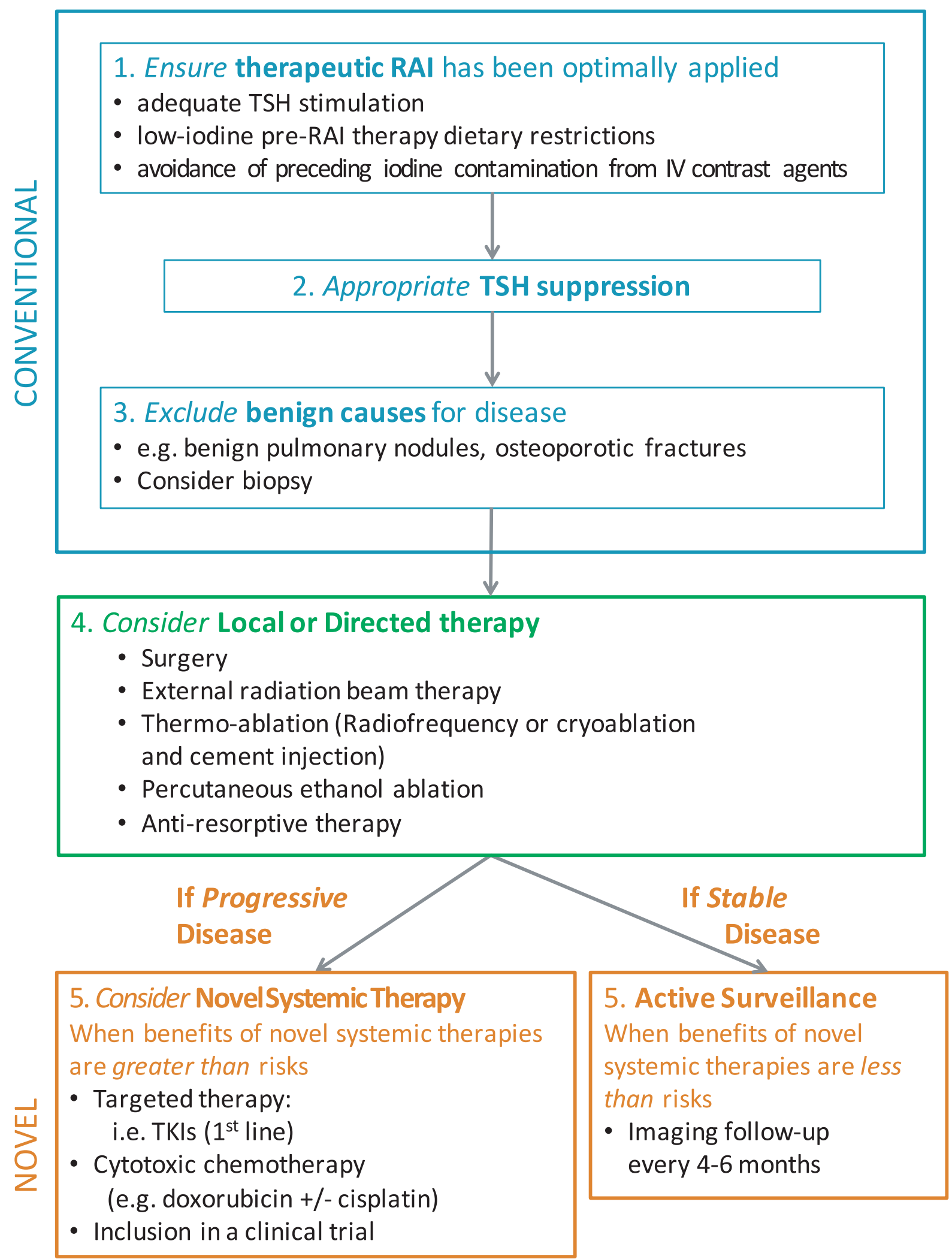

Figure 5

Management approach to radioiodine-refractory differentiated thyroid cancer (adapted from $(2,4)$ ). RAl, radioactive iodine; TKI, tyrosine kinase inhibitor. 
Table 1 Class-related adverse effects of tyrosine kinase inhibitors may be considered as either common or serious (2).

\begin{tabular}{ll}
\hline Common & Serious \\
\cline { 1 - 1 } Diarrhoea & Thrombosis \\
Fatigue & Bleeding \\
Induced hypertension & Heart failure \\
Hepatotoxicity & Hepatotoxicity \\
Skin changes & Gl tract fistula formation \\
Nausea & Intestinal perforation \\
Increased thyroxine dosage & \\
requirement & \\
Changes in taste & \\
Weight loss & \\
\hline
\end{tabular}

Sorafenib was the first TKI approved for the treatment of patients with thyroid cancer in Australia. It has been shown to increase median progression-free survival (PFS) from 5.8 to 10.8 months in progressive radioiodinerefractory locally advanced or metastatic DTC (6). However, treatment response by RECIST criteria was seen in only $12 \%$ of those treated. To date, this small proportion of responders remains poorly defined and mutation status of proteins within oncogenic signalling pathways such as BRAF or RAS does not predict response (6). Adverse events, mostly mild or moderate, were common and often lead to dose reduction but did not usually require withdrawal from the study.

Lenvatinib was the second TKI to be approved by the US Food and Drug Administration (after sorafenib) for the treatment of patients with progressive radioiodinerefractory locally advanced or metastatic DTC. This was based on a study that demonstrated that it is associated with a median PFS of 18.3 months compared with 3.6 months for placebo (7). The type of adverse effects and discontinuation rates were similar to that of sorafenib.

No head-to-head data exist comparing sorafenib to lenvatinib; however, it is our experience that more impressive responses to lenvatinib are often seen and therefore we would generally consider it first line if available. Although we do acknowledge that lenvatinib is significantly more expensive; in Australia, drug price is currently not an obstacle as lenvatinib is subsidised by Medicare Australia for this indication, and sorafenib is available at minimal cost to patients via a drug familiarisation program from the manufacturer.

The issue of biomarkers is important and an area of current research but has not yet been introduced into routine clinical practice. It is of interest however that, for instance, a combination of RAS and BRAF mutation with baseline VEGF and ANG-2 or treatment-associated changes in FGF-2 and IL-10 level has been found to correlate with lenvatinib treatment response (8) so that it seems likely that biomarker profiling will be used to assist therapeutic choice in the future.

The approach to TKI use consists of pre-initiation assessment followed by close monitoring $(4,9)$. Preinitiation assessment involves thorough counselling on potential benefits and adverse effects as well as consideration for alternative approaches including supportive care. Baseline organ function should be documented with particular attention to cardiovascular, hepatic and renal status. Suggested follow-up schedule consists of clinical and laboratory assessment every 2 weeks for the first month, and then monthly up to the third month and then three-monthly thereafter (4). Assessment should focus on blood pressure, skin, bone marrow, hepatic, renal and cardiac function (2). Imaging to assess for disease progression may be considered every one to three months depending on disease trajectory prior to TKI initiation. The clinician must consider the increased risk of haemorrhage, thrombosis and impaired wound healing should concomitant interventions be required. If symptoms occur that may be attributable to focal metastatic disease progression concomitant local therapies should be considered.

Hand-foot skin reactions are the most common adverse effect (6). Like many of the other common class-related adverse effects management includes dose reduction or treatment interruption if quality of life is sufficiently impaired despite supportive care (e.g. topical therapies). Keratoacanthoma-type squamous cell carcinoma is another well-described adverse effect. These atypical lesions can develop within weeks and may occur in non-sun-exposed areas but do not metastasise. Management is excision.

TKI therapy should continue as long as the net benefit outweigh adverse effects (2). In the case of sorafenib, this may be for at least one year given this is the typical duration of response (4) but in up to $20 \%$ of patients may be less due to early discontinuation due to adverse effects (6). Other TKIs may be effective as 'salvage therapy' even after treatment failure occurs on the first agent (10). This could be due to the numerous different targets these agents inhibit with additional actions of a second agent allowing resistance to be overcome. Overall survival with TKI therapy remains undefined as opposed to progressionfree survival due to the cross-over trial construction but appears to be prolonged based on rank-preserving structural failure time (RPSFT) analysis of follow-up trial data (11). 


\section{Declaration of interest}

D J T was a principal site investigator for the SELECT trial of lenvatinib in advancing non-iodine avid differentiated thyroid cancer. He received no personal remuneration.

\section{Funding}

This research did not receive any specific grant from any funding agency in the public, commercial or not-for-profit sector.

\section{Patient consent}

Written consent for the publication of this case report was obtained from the patient.

\section{Author contribution statement}

D J T is the physician responsible for the patient. S D C is the primary author of the manuscript and D J T provided further intellectual input into the manuscript.

\section{References}

1 Durante C, Haddy N, Baudin E, Leboulleux S, Hartl D, Travagli JP, Caillou B, Ricard M, Lumbroso JD, De Vathaire F, et al. 2006 Longterm outcome of 444 patients with distant metastases from papillary and follicular thyroid carcinoma: benefits and limits of radioiodine therapy. Journal of Clinical Endocrinology and Metabolism 91 2892-2899. (doi:10.1210/jc.2005-2838)

2 Haugen BR, Alexander EK, Bible KC, Doherty GM Mandel SJ, Nikiforov YE, Pacini F, Randolph GW, Sawka AM, Schlumberger M, et al. 20152015 American Thyroid Association Management Guidelines for adult patients with thyroid nodules and differentiated thyroid cancer: the American Thyroid Association Guidelines task force on thyroid nodules and differentiated thyroid cancer. Thyroid 26 1-133. (doi:10.1089/thy.2015.0020)

3 Haugen BR \& Sherman SI 2013 Evolving approaches to patients with advanced differentiated thyroid cancer. Endocrine Reviews 34 439-455. (doi:10.1210/er.2012-1038)
4 Klopper J \& Haugen BR 2014 S4-New treatment algorithms for systemic therapy in managing aggressive thyroid cancer. In Special Endotext and Thyroid Manager Symposium on Tyrosine Kinase Inhibitors in Management of Aggressive Differentiated Thyroid Cancer. South Dartmouth, MA 02748: ENDOCRINE EDUCATION Inc.

5 Rini BI, Cohen DP, Lu DR, Chen I, Hariharan S, Gore ME, Figlin RA, Baum MS \& Motzer RJ 2011 Hypertension as a biomarker of efficacy in patients with metastatic renal cell carcinoma treated with sunitinib. Journal of the National Cancer Institute 103 763-773. (doi:10.1093/jnci/djr128)

6 Brose MS, Nutting CM, Jarzab B, Elisei R, Siena S, Bastholt L, de la Fouchardiere C, Pacini F, Paschke R, Shong YK, et al. 2014 Sorafenib in radioactive iodine-refractory, locally advanced or metastatic differentiated thyroid cancer: a randomised, double-blind, phase 3 trial. Lancet 384 319-328. (doi:10.1016/S0140-6736(14)60421-9)

7 Schlumberger M, Tahara M, Wirth LJ, Robinson B, Brose MS, Elisei R, Habra MA, Newbold K, Shah MH, Hoff AO, et al. 2015 Lenvatinib versus placebo in radioiodine-refractory thyroid cancer. New England Journal of Medicine 372 621-630. (doi:10.1056/ NEJMoa1406470)

8 Ball DW, Sherman SI, Jarzab B, Cabanillas ME, Martins R, Shah MH, Bodenner D, Newbold K, Licitra LF, Topliss D, et al. 2012 Lenvatinib treatment of advanced RAI-refractory differentiated thyroid cancer (DTC): cytokine and angiogenic factor (CAF) profiling in combination with tumor genetic analysis to identify markers associated with response. Journal of Clinical Oncology $\mathbf{3 0}$ (Supplement); abstr 5518

9 Carhill AA, Cabanillas ME, Jimenez C, Waguespack SG, Habra MA, $\mathrm{Hu}$ M, Ying A, Vassilopoulou-Sellin R, Gagel RF, Sherman SI, et al. 2013 The noninvestigational use of tyrosine kinase inhibitors in thyroid cancer: establishing a standard for patient safety and monitoring. Journal of Clinical Endocrinology and Metabolism 98 31-42. (doi:10.1210/jc.2012-2909)

10 Dadu R, Devine C, Hernandez M, Waguespack SG, Busaidy NL, Hu MI, Jimenez C, Habra MA, Sellin RV, Ying AK, et al. 2014 Role of salvage targeted therapy in differentiated thyroid cancer patients who failed first-line sorafenib. Journal of Clinical Endocrinology and Metabolism 99 2086-2094. (doi:10.1210/jc.2013-3588)

11 Brose MS, Jarzab B, Elisei R, Siena S, Bastholt L, De La Fouchardiere C, Pacini F, Paschke R, Nutting C, Shong YK, et al. 2014 Updated overall survival analysis of patients with locally advanced or metastatic radioactive iodine-refractory differentiated thyroid cancer (RAI-rDTC) treated with sorafenib on the phase 3 DECISION trial. Journal of Clinical Oncology 32 (Supplement); abstr 6060^

Received in final form 24 November 2016

Accepted 12 January 2017 